\title{
Análise da prevalência de doenças cardiovasculares e fatores associados em idosos, 2000-2010
}

\author{
Analysis of the prevalence of cardiovascular diseases \\ and associated factors among the elderly, 2000-2010
}

Kaio Henrique Correa Massa ${ }^{1}$

Yeda Aparecida Oliveira Duarte ${ }^{2}$

Alexandre Dias Porto Chiavegatto Filho ${ }^{3}$

${ }^{1}$ Programa de PósGraduação em Saúde Pública, Faculdade de Saúde Pública (FSP), Universidade de São Paulo (USP). Av.

Dr. Arnaldo 715, Cerqueira César. 05405-000 São Paulo SP Brasil.

kaiomassa@usp.br

${ }^{2}$ Escola de Enfermagem,

USP. São Paulo SP Brasil.

${ }^{3}$ Departamento de

Epidemiologia, FSP, USP.

São Paulo SP Brasil.

\begin{abstract}
The scope of this study was to analyze the difference in the prevalence of cardiovascular disease (CVD) between 2000 and 2010 and its association with socio-economic and risk factors among the elderly. Bayesian multilevel models were applied to analyze the difference in the prevalence of CVD, and the association between individual characteristics and the presence of CVD was analyzed by logistic regression models with complex samples separately for 2000, 2006 and 2010. This research is part of the Health, Welfare and Aging (SABE) study, performed in Sao Paulo, Brazil, and used the samples collected in 2000, 2006 and 2010. An increase in the prevalence of cardiovascular disease among the elderly was observed during the period, from $17.9 \%$ in 2000 , to $22.2 \%$ in 2006 , attaining $22.9 \%$ in 2010 . In reference to the prevalence observed in 2000, the multilevel analysis indicated a significant increase in the presence of cardiovascular disease in 2006 $(\mathrm{OR}=3.2095 \% \mathrm{CI}=1.93-5.31)$ and $2010(\mathrm{OR}$ $=2.9895 \% C I=1.51-5.89)$, even after statistical adjustment for individual factors. The presence of CVD was associated with older age, smoking history, the presence of diabetes and hypertension, and an inverse association was observed between the presence of CVD and alcohol intake.

Key words Cardiovascular diseases, Epidemiology, Elderly, Chronic disease
\end{abstract}

Resumo O objetivo do estudo foi analisar a mudança na prevalência de doença cardiovascular (DCV) entre 2000 e 2010 e sua associação com os fatores socioeconômicos e fatores de risco em idosos. A diferença da prevalência de DCV ao longo do período foi analisada por meio de modelos multinível bayesianos e a análise da associação entre a presença de DCV e os fatores individuais utilizou modelos de regressão logística para amostras complexas nos três periodos separadamente (2000, 2006 e 2010). A presente pesquisa utilizou os dados do Estudo de Saúde, Bem-Estar e Envelhecimento (SABE), realizada no município de São Paulo, referente às amostras de 2000, 2006 e 2010. Foi observado um aumento geral na prevalência de DCV em idosos no município de São Paulo na última década, apresentando prevalências iguais a 17,9\% em 2000,22,2\% em 2006 e 22,9\% em 2010. Em relação à prevalência em 2000, foi observado o aumento estatisticamente significativo da presença de DCV em 2006 (OR $=3,20$ IC95\% = 1,93-5,31) e $2010(\mathrm{OR}=2,98$ IC95\% $=1,51-5,89)$, mesmo após o ajuste estatístico para características individuais. A presença de DCV apresentou associação com maior faixa etária, histórico de tabagismo e presença de diabetes e hipertensão arterial, sendo observada uma associação inversa entre a presença de DCV e a ingestão de álcool.

Palavras-chave Doenças cardiovasculares, Epidemiologia, Idoso, Doença crônica 


\section{Introdução}

O aumento no número de anos vividos com alguma doença crônica ${ }^{1}$, principalmente entre indivíduos mais velhos ${ }^{2}$, tem se tornado uma das principais preocupações em saúde pública. Nesse contexto, destaca-se a morbidade por doenças cardiovasculares (DCV), que é considerada por estudos recentes de carga de doença como a principal responsável por anos de vida perdidos ajustados por incapacidade em idosos ${ }^{3}$.

Segundo a Organização Mundial de Saúde (OMS), observa-se um aumento recente na carga de doenças cardiovasculares, principalmente em países de baixa e média rendas ${ }^{4}$, reflexo do aumento da expectativa de vida e, consequentemente, do maior tempo de exposição aos fatores risco para as doenças crônicas não transmissíveis $(\mathrm{DCNT})^{5}$. As doenças cardiovasculares são atualmente a principal causa de morte nos países em desenvolvimento ${ }^{6}$, e espera-se que continue sendo a causa de mortalidade mais importante no mundo durante a próxima década ${ }^{7}$.

No Brasil, as doenças cardiovasculares são responsáveis por $27,7 \%$ dos óbitos, atingindo $31,8 \%$ quando são excluídos os óbitos por causa externas $^{8}$, sendo consideradas a principal causa de morte ${ }^{9}$. Embora tenha sido observada uma recente redução da sua presença como causa de mortalidade ${ }^{10,11}$, o mesmo não pode se afirmar a respeito da morbidade por DCV, considerada o fator de maior impacto no custo das internações hospitalares no país ${ }^{10}$. Em 2014, 10,1\% das internações no Brasil foram causadas por doenças do aparelho circulatório, e, do total dessas internações, 57,2\% foram entre indivíduos de 60 anos ou mais ${ }^{8}$.

A associação entre a longevidade e o aumento de doenças crônicas é conhecida na literatura ${ }^{5,12}$ e os resultados da Pesquisa Nacional de Saúde (PNS) apontam para uma maior morbidade por doenças cardiovasculares em indivíduos mais ve$\operatorname{lhos}^{13}$. Segundo a PNS, em 2013 a prevalência das doenças cardiovasculares na população adulta brasileira ( $\geq 18$ anos) era de $4,2 \%$, apresentando um gradiente crescente nos grupos de maior idade da população, sendo a prevalência de DCV entre idosos de $11,4 \%{ }^{13}$.

A avaliação da carga das doenças crônicas na população representa um importante campo na saúde pública e no planejamento da atenção em saúde ${ }^{3}$. Entretanto, estudos que analisaram a mudança na prevalência de doenças cardiovasculares ainda são escassos no Brasil e a avaliação de mudanças no quadro epidemiológico da DCV na última década pode contribuir para uma visão atualizada da situação de saúde dos idosos, além de trazer à luz novos comparativos para os dados existentes na literatura, analisando a relação entre a morbidade por DCV, os fatores socioeconômicos, comportamentais e a presença de doenças crônicas. Assim, o presente estudo tem como objetivo analisar a mudança na morbidade por doenças cardiovasculares entre 2000 e 2010 em idosos residentes no município de São Paulo. Pretende-se também analisar os fatores associados à prevalência de doenças cardiovasculares ao longo do período.

\section{Metodologia}

A presente análise é parte do Estudo de Saúde, Bem-Estar e Envelhecimento (SABE), uma pesquisa de múltiplas coortes que coletou dados de amostras representativas dos idosos residentes no município de São Paulo, nos anos 2000, 2006 e 2010. O presente trabalho é um estudo longitudinal seriado que teve como objetivo analisar a diferença na prevalência das doenças cardiovasculares ao longo do período e a associação das características sociodemográficas, econômicas e fatores de risco associados com a presença de doenças cardiovasculares em cada um dos três anos de coleta.

Os dados foram obtidos por entrevistas domiciliares, utilizando um questionário que coletou informações sobre dados pessoais, condições de saúde, dados antropométricos, estado funcional, uso de medicamentos, história laboral, além de uso e acesso a serviços e características de moradia. O Estudo SABE é coordenado pelo Departamento de Epidemiologia da Faculdade de Saúde Pública da Universidade de São Paulo, tendo sido aprovado pelo Comitê de Ética em Pesquisa da Faculdade de Saúde Pública da Universidade de São Paulo e pelo Comitê Nacional de Ética em Pesquisa (Conep) $)^{14}$.

O estudo foi composto por uma amostra probabilística, estratificada por sexo e faixa etária, utilizando o método de amostra por conglomerado sob o critério de probabilidade proporcional ao número de domicílios, realizada a partir do sorteio de domicílios baseado no cadastro permanente de setores censitários da Pesquisa Nacional por Amostra de Domicílios (PNAD) ${ }^{14}$. $\mathrm{O}$ estudo contou ainda com uma sobreamostra compensatória da mortalidade na população com 75 anos e mais com o objetivo de completar o número desejado de entrevistas para esta faixa etária ${ }^{14}$. 
A variável dependente utilizada pelo presente estudo foi a presença de doença cardiovascular, obtida pela resposta positiva do indivíduo à pergunta: "Alguma vez um médico ou enfermeiro lhe disse que o(a) $\mathrm{Sr}(\mathrm{a})$ teve um ataque do coração, uma doença coronária, angina, doença congestiva ou outros problemas cardíacos?". As variáveis relacionadas às informações demográficas e socioeconômicas foram sexo, idade em anos (categorizada nas faixas 60 a 64, 65 a 69, 70 a 74, 75 a 79, 80 anos ou mais), cor da pele (nas categorias branca, parda, preta e outras), escolaridade (em anos completos de estudo formal), renda per capita do entrevistado (em número de salários mínimos referentes ao ano da coleta) e situação marital (categorizada em viver sem ou com companheiro).

As análises também incluíram características comportamentais e fatores de risco, especificamente, o consumo de álcool, o histórico de tabagismo e o índice de massa corpórea (IMC). Os dados sobre a ingestão de bebidas alcoólicas foram obtidos pelo recordatório dos últimos três meses precedentes à entrevista. $\mathrm{O}$ histórico de tabagismo foi dividido em três categorias: nunca fumou, já fumou e fuma atualmente. O IMC foi calculado utilizando as medidas de peso e a altura de cada indivíduo $\left(\mathrm{Kg} / \mathrm{m}^{2}\right)$, aferidas por profissionais devidamente treinados em visita domiciliar, e, posteriormente, categorizado em baixo peso $\left(<22 \mathrm{~kg} / \mathrm{m}^{2}\right)$, peso adequado $(\geq 22 \mathrm{e}$ $\left.<27 \mathrm{~kg} / \mathrm{m}^{2}\right)$ e sobrepeso $\left(\geq 27 \mathrm{~kg} / \mathrm{m}^{2}\right)$, segundo preconizado pela Norma Técnica da Vigilância Alimentar e Nutricional para valores referentes a idosos ${ }^{15}$.

Foram incluídas também nas características individuais de análise a presença de comorbidades, mais especificamente, diabetes tipo 2 e hipertensão arterial. Ambas informações foram obtidas pelo autorrelato do entrevistado sobre ter sido informado por algum profissional médico ou enfermeiro de ser portador de alguma das duas doenças.

Inicialmente, foram utilizados modelos de regressão logística para analisar a associação da presença de doenças cardiovasculares com as caraterísticas socioeconômicas, fatores de risco e a presença de comorbidades em 2000, 2006 e 2010, separadamente, considerando a ponderação necessária para a análise de amostras complexas. As análises multivariadas utilizaram ajuste estatístico para os fatores inseridos no modelo, seguindo a estrutura conceitual descrita por Victora et al. ${ }^{16}$, tendo como ordem de hierarquização as características demográficas, seguidas das socioeconômicas e dos fatores de risco.
Para avaliar a qualidade do ajuste nas estimativas dos parâmetros, os modelos multivariados foram testados quanto a multicolinearidade das variáveis independentes utilizando-se o VIF (Variance Inflation Factor), que indica quanto a variância dos coeficientes está inflada comparada às covariáveis do modelo de regressão, não sendo observada multicolinearidade entre as variáveis que integraram os modelos de análise.

A análise da diferença da prevalência de doenças cardiovasculares entre 2000, 2006 e 2010 utilizou um modelo de regressão logística multinível, considerando a não independência das observações devido ao caráter longitudinal do Estudo SABE, em que os indivíduos das coortes anteriores são revisitados, havendo reposição apenas da população de 60 a 64 anos nas ondas subsequentes. A análise da diferença da presença de DCV entre os períodos foi realizada pela introdução de variáveis dummy de ano no modelo com os dados agregados dos três períodos, sendo considerado como primeiro nível o período de análise (2000, 2006 e 2010) e o segundo nível composto pelos indivíduos e suas caraterísticas.

Para estimar os parâmetros do modelo foi utilizada inferência bayesiana, uma abordagem recomendada para diminuir o viés em análises multinível com variáveis dependentes dicotômicas $^{17}$, além de permitir a comparação da qualidade de ajuste dos modelos, utilizando o Bayesian Information Criterion (BIC), no qual a diminuição dos valores do coeficiente indica melhor ajuste do modelo ${ }^{17}$.

As análises foram realizadas no programa Stata 13.1 (Stata Corporation, College Station, Texas, 2013), utilizando para as análises descritivas e multivariadas separadas por ano o modo survey, que considera o procedimento complexo da amostra: pesos amostrais e organização dos indivíduos (unidades amostrais secundárias) nos setores censitários da cidade (unidades amostrais primárias), e para a análise agregada o comando gllamm, que permite a análise estatística considerando a estrutura multinível dos dados e a inclusão da ponderação necessária para a análise de amostras complexas. Para todas as análises, foi utilizado nível de significância de $\mathrm{p} \leq 0,05$.

\section{Resultados}

As amostras foram constituídas por 2.143, 1.413, 1.333 indivíduos com idade igual ou superior a 60 anos, de ambos os sexos, residentes do município de São Paulo, em 2000, 2006 e 2010, respec- 
tivamente. A perda amostral observada ao longo das coletas foi investigada e, embora não tenha afetado a representatividade da população nas amostras, foi identificado como principal determinante os óbitos ocorridos no período, confirmados por linkage nas bases de dados de óbitos estaduais e municipais.

Nos três períodos, a maioria dos idosos era do sexo feminino (58,8\% em 2000, 59,4\% em 2006 e $59,9 \%$ em 2010). O relato de cor de pele mais frequente ao longo do período foi a cor de pele branca $(70,4 \%$ em 2000, 63,5\% em 2006 e 58,7\% em 2010), seguido pelo autorrelato de cor de pele parda. Em relação à escolaridade, foi observada uma diminuição dos indivíduos com nenhuma ou pouca escolaridade ao longo dos anos de estudo, sendo que o maior aumento observado na escolaridade foi entre aqueles com oito ou mais anos de escolaridade. A maioria dos indivíduos apresentou renda menor que três salários mínimos ao longo do período, chamando atenção a acentuada diminuição de idosos que relataram receber menos de um salário mínimo nos últimos anos de seguimento. Em relação à situação marital, a maioria relatou viver com companheiro em todos os três períodos de coleta (Tabela 1).

Em relação às características comportamentais e fatores de risco no período como um todo, cerca de $70 \%$ dos idosos relataram não terem ingerido álcool no recordatório de três meses anteriores à entrevista. Em relação ao tabagismo, a maioria também relatou nunca ter fumado, sendo observado uma discreta diminuição entre aqueles que fumam atualmente $(15,8 \%$ em $2000,14,0 \%$ em 2006 e 12,0\% em 2010). A classificação segundo índice de massa corpórea mostrou uma baixa proporção de idosos considerados de baixo peso e um constante acréscimo entre os indivíduos com sobrepeso na última década $(40,9 \%$ em 2000 , 43,0\% em 2006 e 55,1\% em 2010) (Tabela 1).

A presença de morbidades, sem exceção, também apresentou um aumento ao longo do período. A prevalência de diabetes atingiu mais de um quarto da população de idosos residentes no município de São Paulo, em 2010, especificamente aumentando de $16,7 \%$, em 2000 , para $21,1 \%$, em 2006, e alcançando 25,2\%, em 2010. Em relação à hipertensão arterial, o aumento foi ainda mais acentuado; a prevalência em 2000 era 53,1\%, passando para $62,7 \%$, em 2006 , e atingindo $66,8 \%$ dos idosos, em 2010. As doenças cardiovasculares seguiram a mesma tendência, apresentando prevalências iguais a $17,9 \%, 22,2 \%$ e $22,9 \%$, em 2000, 2006 e 2010, respectivamente (Tabela 1).
Em relação às análises de regressão da presença de doenças cardiovasculares e fatores associados em 2000, 2006 e 2010, analisadas de forma separada, foram observadas associações estatisticamente significativas entre as doenças cardiovasculares com a idade, situação marital, ingestão de álcool, tabagismo, diabetes e hipertensão arterial. Foi observado uma associação entre a presença de DCV com as faixas etárias mais elevadas nos três períodos. Em relação à situação marital de viver sem companheiro, foi observada uma razão de chances menor da presença de DCV apenas em 2010 (OR = 0,66 IC95\%=0,48-0,91). A ingestão de álcool apresentou associação inversa com a morbidade de DCV, em 2000 e 2010 (OR $=0,63 \mathrm{IC} 95 \%=0,44-0,92 ; \mathrm{OR}=0,68 \mathrm{IC} 95 \%=$ 0,47-0,98, respectivamente). O histórico de tabagismo esteve associado com maior razão de chances de DCV (OR = 1,45 IC95\% = 1,05-2,00 em 2000 e OR $=1,49$ IC95\% $=1,04-2,13 \mathrm{em}$ 2010) (Tabela 2).

A presença de diabetes e hipertensão arterial apresentaram associações significativas com a presença de doenças cardiovasculares. Em relação à presença de diabetes, foi observada maior razão de chances estatisticamente significativas da presença de DCV em 2006 e 2010 (OR = 1,58 IC95\% = 1,05-2,36; OR = 1,90 IC95\% = 1,342,70, respectivamente). Quanto à hipertensão arterial, observou-se aumento da chance de DCV nos três períodos $(\mathrm{OR}=2,80 \mathrm{IC} 95 \%=1,92-4,08$, em 2000; OR $=2,92$ IC95\% $=2,02-4,21$, em 2006; OR = 2,22 IC95\% = 1,44-3,44, em 2010) (Tabela 2).

A análise multinível das informações agregadas teve como objetivo verificar a existência de diferenças significativas entre a prevalência de doenças cardiovasculares, entre 2000, 2006 e 2010, e foi realizada em dois modelos. O primeiro procurou analisar as diferenças, em 2006 e 2010, tendo como referência a presença de DCV, em 2000. E um segundo utilizando a informação de 2006 como referência, a fim de comparar com a onda subsequente, 2010. Em relação a 2000, foi observado um aumento estatisticamente significativo da razão de chances de DCV, em 2006 e 2010 (OR $=3,20$ IC95\% $=1,93-5,31 ;$ OR = 2,98 IC95\% = 1,51-5,89, respectivamente), mesmo após o controle pelas variáveis individuais. No segundo modelo, foi observado um aumento na presença de DCV apenas na análise bruta, não sendo verificada associação estatisticamente significativa após a introdução das variáveis com as características individuais no modelo ajustado (Tabela 3 ). 
Tabela 1. Distribuição dos idosos segundo características demográficas, socioeconômicas e fatores de risco nos segmentos 2000, 2006 e 2010. Município de São Paulo, Brasil.

\begin{tabular}{|c|c|c|c|c|c|c|}
\hline & \multicolumn{2}{|c|}{$2000(n=2143)^{*}$} & \multicolumn{2}{|c|}{$2006(n=1413)^{*}$} & \multicolumn{2}{|c|}{$2010(n=1333)^{*}$} \\
\hline & $n^{1}$ & $\%^{2}$ & $\mathbf{n}^{1}$ & $\%^{2}$ & $\mathbf{n}^{1}$ & $\%^{2}$ \\
\hline \multicolumn{7}{|l|}{ Sexo } \\
\hline Homem & 878 & 41,2 & 540 & 40,6 & 477 & 40,1 \\
\hline Mulher & 1265 & 58,8 & 873 & 59,4 & 856 & 59,9 \\
\hline \multicolumn{7}{|l|}{ Idade } \\
\hline $60-64$ & 426 & 32,3 & 298 & 31,0 & 355 & 31,6 \\
\hline $65-69$ & 379 & 26,8 & 237 & 27,7 & 231 & 22,6 \\
\hline $70-74$ & 336 & 18,8 & 208 & 17,7 & 218 & 17,7 \\
\hline $75-79$ & 472 & 11,2 & 232 & 12,4 & 166 & 12,8 \\
\hline $80 \mathrm{ou}+$ & 530 & 10,9 & 438 & 11,2 & 363 & 15,3 \\
\hline \multicolumn{7}{|l|}{ Cor da pele } \\
\hline Branca & 1524 & 70,4 & 930 & 63,5 & 775 & 58,7 \\
\hline Parda & 420 & 21,2 & 258 & 20,2 & 383 & 29,3 \\
\hline Preta & 86 & 3,9 & 101 & 7,3 & 94 & 6,7 \\
\hline Outras & 101 & 4,5 & 122 & 9,0 & 68 & 5,3 \\
\hline \multicolumn{7}{|l|}{ Escolaridade } \\
\hline $0-3$ anos & 1074 & 45,2 & 660 & 42,2 & 527 & 35,3 \\
\hline $4-7$ anos & 730 & 36,8 & 514 & 38,6 & 494 & 37,3 \\
\hline 8 anos ou + & 327 & 18,0 & 235 & 19,2 & 311 & 27,4 \\
\hline \multicolumn{7}{|c|}{ Renda (em salários mínimos) } \\
\hline Menos de 1 & 777 & 37,5 & 363 & 26,1 & 41 & 3,6 \\
\hline $1-3$ & 338 & 14,1 & 347 & 26,6 & 624 & 49,8 \\
\hline $3-5$ & 431 & 21,0 & 345 & 27,2 & 321 & 27,5 \\
\hline $5 \mathrm{ou}+$ & 497 & 27,4 & 235 & 20,1 & 180 & 19,1 \\
\hline \multicolumn{7}{|l|}{ Situação marital atual } \\
\hline Com companheiro & 1122 & 57,1 & 694 & 57,4 & 659 & 55,0 \\
\hline Sem companheiro & 1020 & 42,9 & 717 & 42,6 & 658 & 45,0 \\
\hline \multicolumn{7}{|c|}{ Ingestão de álcool nos últimos três meses } \\
\hline Não & 1514 & 68,3 & 1046 & 69,2 & 951 & 68,2 \\
\hline Sim & 629 & 31,7 & 363 & 30,8 & 381 & 31,8 \\
\hline \multicolumn{7}{|l|}{ Tabagismo } \\
\hline Nunca fumou & 1155 & 52,1 & 770 & 52,0 & 702 & 51,0 \\
\hline Já fumou & 697 & 32,1 & 486 & 34,0 & 488 & 37,0 \\
\hline Fuma atualmente & 290 & 15,8 & 157 & 14,0 & 142 & 12,0 \\
\hline \multicolumn{7}{|l|}{ IMC } \\
\hline Baixo peso & 312 & 15,1 & 231 & 15,2 & 169 & 11,2 \\
\hline Peso adequado & 770 & 44,0 & 555 & 41,8 & 425 & 33,7 \\
\hline Sobrepeso & 714 & 40,9 & 537 & 43,0 & 650 & 55,1 \\
\hline \multicolumn{7}{|l|}{ Diabetes } \\
\hline Não & 1769 & 83,3 & 1114 & 78,9 & 998 & 74,8 \\
\hline Sim & 358 & 16,7 & 292 & 21,1 & 333 & 25,2 \\
\hline \multicolumn{7}{|l|}{ Hipertensão } \\
\hline Não & 985 & 46,9 & 506 & 37,3 & 432 & 33,2 \\
\hline Sim & 1143 & 53,1 & 904 & 62,7 & 900 & 66,8 \\
\hline \multicolumn{7}{|c|}{ Doenças cardiovasculares } \\
\hline Não & 1706 & 82,1 & 1050 & 77,8 & 1009 & 77,1 \\
\hline Sim & 425 & 17,9 & 350 & 22,2 & 322 & 22,9 \\
\hline
\end{tabular}


Tabela 2. Modelos de regressão logística para presença de doenças cardiovasculares ajustadas segundo características demográficas, socioeconômicas e fatores de risco em idosos, analisados separadamente para 2000, 2006 e 2010. Município de São Paulo, Brasil.

\begin{tabular}{|c|c|c|c|c|c|c|}
\hline & \multicolumn{2}{|c|}{$2000^{1}$} & \multicolumn{2}{|c|}{$2006^{1}$} & \multicolumn{2}{|c|}{$2010^{1}$} \\
\hline & OR & IC $95 \%$ & OR & IC 95\% & OR & IC $95 \%$ \\
\hline \multicolumn{7}{|l|}{ Sexo } \\
\hline Mulher & 0,84 & $0,67-1,08$ & 0,80 & $0,59-1,07$ & 1,06 & $0,82-1,36$ \\
\hline \multicolumn{7}{|l|}{ Idade } \\
\hline $65-69$ & 1,37 & $0,89-2,11$ & 1,40 & $0,82-2,37$ & 1,64 & $0,92-2,95$ \\
\hline $70-74$ & $1,89^{* *}$ & $1,26-2,83$ & 1,36 & $0,76-2,44$ & $1,92^{*}$ & $1,17-3,15$ \\
\hline $75-79$ & $1,92^{* *}$ & $1,38-2,66$ & $1,71^{*}$ & $1,02-2,86$ & $1,94^{*}$ & $1,15-3,25$ \\
\hline 80 e mais & $2,24^{* *}$ & $1,62-3,08$ & $2,32^{* *}$ & $1,43-3,76$ & $2,91^{* *}$ & $1,88-4,52$ \\
\hline \multicolumn{7}{|l|}{ Cor da pele } \\
\hline Parda & 0,76 & $0,55-1,07$ & 1,04 & $0,68-1,58$ & 0,98 & $0,69-1,39$ \\
\hline Preta & 1,01 & $0,54-1,86$ & 1,46 & $0,83-2,57$ & 1,40 & $0,79-2,48$ \\
\hline Outras & 0,65 & $0,32-1,31$ & 1,13 & $0,60-2,13$ & 1,02 & $0,54-1,94$ \\
\hline \multicolumn{7}{|l|}{ Escolaridade } \\
\hline $4-7$ anos & 0,74 & $0,55-1,01$ & 0,98 & $0,65-1,49$ & 0,98 & $0,66-1,46$ \\
\hline 8 anos ou + & 0,92 & $0,61-1,38$ & 0,65 & $0,37-1,13$ & 0,70 & $0,43-1,15$ \\
\hline \multicolumn{7}{|c|}{ Renda (em salários mínimos) } \\
\hline $1-3$ & 0,97 & $0,66-1,41$ & 1,33 & $0,86-2,07$ & 1,17 & $0,48-2,87$ \\
\hline $3-5$ & 0,91 & $0,66-1,25$ & 1,06 & $0,66-1,68$ & 1,16 & $0,49-2,76$ \\
\hline $5 \mathrm{ou}+$ & 1,14 & $0,79-1,65$ & 1,68 & $0,98-2,88$ & 1,38 & $0,48-3,95$ \\
\hline \multicolumn{7}{|l|}{ Situação marital atual } \\
\hline Sem companheiro & 1,02 & $0,76-1,37$ & 0,74 & $0,49-1,12$ & $0,66^{*}$ & $0,48-0,91$ \\
\hline \multicolumn{7}{|c|}{ Ingestão de álcool nos últimos três meses } \\
\hline Sim & $0,63^{*}$ & $0,44-0,92$ & 0,80 & $0,54-1,18$ & $0,68^{*}$ & $0,47-0,98$ \\
\hline \multicolumn{7}{|l|}{ Tabagismo } \\
\hline Já fumou & $1,45^{*}$ & $1,05-2,00$ & 1,49 & $0,97-2,28$ & $1,49^{*}$ & $1,04-2,13$ \\
\hline Fuma atualmente & 1,31 & $0,84-2,03$ & 0,92 & $0,50-1,67$ & 0,80 & $0,44-1,43$ \\
\hline \multicolumn{7}{|l|}{ IMC } \\
\hline Peso adequado & 1,01 & $0,65-1,58$ & 1,11 & $0,65-1,89$ & 1,37 & $0,79-2,36$ \\
\hline Sobrepeso & 1,26 & $0,80-1,99$ & 1,35 & $0,80-2,28$ & 1,57 & $0,96-2,56$ \\
\hline \multicolumn{7}{|l|}{ Diabetes } \\
\hline Sim & 1,01 & $0,68-1,49$ & $1,58^{*}$ & $1,05-2,36$ & $1,90^{* *}$ & $1,34-2,70$ \\
\hline \multicolumn{7}{|l|}{ Hipertensão } \\
\hline Sim & $2,80^{* *}$ & $1,92-4,08$ & $2,92^{* *}$ & $2,02-4,21$ & $2,22^{* *}$ & $1,44-3,44$ \\
\hline
\end{tabular}

\section{Discussão}

O presente estudo analisou a mudança na prevalência de doenças cardiovasculares em idosos ao longo da última década (2000 a 2010), além de ter permitido testar a sua associação com fatores socioeconômicos, comportamentais e a presença de comorbidades no município de São Paulo. Foi possível observar que, mesmo após o ajuste estatístico para as variáveis socioeconômicas e fatores de risco, houve aumento estatisticamente significante na prevalência de doenças cardiovas- culares em 2006 e 2010, em relação aos valores observados em 2000.

A associação entre o avanço da idade e o aumento progressivo na prevalência de doenças cardiovasculares foi observado em todas as análises. O envelhecimento está relacionado ao aumento do risco de apresentar doenças crônicas ${ }^{3,18}$, como a DCV, uma vez que o aumento na longevidade acarreta naturalmente em um maior período de exposição a fatores de risco para $\mathrm{DCNT}^{5}$. Dessa forma, a maior presença de DCV nas parcelas mais envelhecidas da população encontradas 
Tabela 3. Modelos logísticos multinível da presença de doenças cardiovasculares ajustados segundo características socioeconômicas, comportamentais e presença de comorbidades em idosos, analisado de forma agregada para 2000, 2006 e 2010. Município de São Paulo, Brasil.

\begin{tabular}{|c|c|c|c|c|}
\hline & \multicolumn{2}{|c|}{ Modelo Bruto $(n=4862)$} & \multicolumn{2}{|c|}{ Modelo Ajustado $(n=3905)$} \\
\hline & OR & IC 95\% & OR & IC $95 \%$ \\
\hline Ano (ref: 2000) & & & & 1,00 \\
\hline 2006 & $5,44^{* *}$ & $3,45-8,59$ & $3,20^{* *}$ & $1,93-5,31$ \\
\hline 2010 & $7,81^{* *}$ & $4,46-13,68$ & $2,98^{*}$ & $1,51-5,89$ \\
\hline \multirow[t]{3}{*}{$\mathrm{BIC}$} & & 3096123 & & 2361444 \\
\hline & \multicolumn{2}{|c|}{ Modelo Bruto $(\mathrm{n}=2731)$} & \multicolumn{2}{|c|}{ Modelo Ajustado $(n=2238)$} \\
\hline & OR & IC 95\% & OR & IC $95 \%$ \\
\hline Ano (ref: 2006) & & & & 1,00 \\
\hline 2010 & $1,50^{*}$ & $1,06-2,12$ & 0,96 & $0,59-1,56$ \\
\hline BIC & & 2335160 & & 1808116 \\
\hline
\end{tabular}

${ }^{*} \mathrm{p}<0,05 ;{ }^{* *} \mathrm{p} \leq 0,001$.

pelo estudo corroboram os resultados anteriores sobre a relação entre o envelhecimento populacional e o aumento das doenças crônicas ${ }^{3,19}$. Nesse contexto, as associações encontradas reforçam a necessidade de um planejamento específico para a atenção das parcelas mais envelhecidas da população $0^{20}$, dado o importante crescimento esperado para os próximos anos dessas faixas etárias $^{21}$.

O aumento da escolaridade observada no período reflete as transformações ocorridas nas características socioeconômicas da população idosa na última década. A diminuição dos indivíduos com baixa ou nenhuma escolaridade é um importante aspecto também relacionado à saúde e, embora não tenham sido observadas associações significativas entre DCV e escolaridade, indivíduos com menor grau de instrução tendem a apresentar pior percepção do estado de saúde ${ }^{22} \mathrm{e}$ maior presença de doenças crônicas ${ }^{23}$.

Durante o processo de envelhecimento, fatores comportamentais, como o tabagismo e o consumo excessivo de álcool, podem levar a disfunções no organismo, aumentando a chance do aparecimento de novas doenças ${ }^{24}$. Em relação ao uso de álcool, verificou-se uma menor razão de chances de DCV associada à ingestão de álcool por idosos, com a exceção do segmento de 2006. O efeito benéfico da ingestão de álcool relacionado às doenças cardiovasculares foi recentemente descrito por Ronksley et al..$^{25} \mathrm{em}$ uma revisão sistemática e meta-análise que apontou para uma redução de múltiplos desfechos cardiovasculares associada à ingestão leve e moderada de álcool. Entretanto, mesmo que aponte um mesmo sentido de associação, a interpretação dos achados do presente estudo deve levar em consideração a impossibilidade de se estabelecer uma relação causal entre a resposta positiva da ingestão de álcool nos últimos três meses e a menor presença de DCV com a presente amostra.

A análise da associação dos fatores de risco modificáveis em relação a presença de doenças cardiovasculares, também permitiu verificar uma associação estatisticamente significativa entre o histórico de tabagismo e a presença de doenças cardiovasculares, com a exceção do período de 2006. Não foram observadas associações entre o tabagismo atual e a presença de DCV, fato que pode indicar uma menor preocupação de cuidados com a saúde e procura por serviços e diagnósticos de doenças, uma vez que o tabagismo é reconhecidamente um dos mais importantes fatores de risco modificáveis relacionados às doenças cardiovasculares ${ }^{4}$.

Durante o período analisado verificou-se um importante aumento dos indivíduos em situação de sobrepeso, um fenômeno mundialmente observado nas últimas décadas ${ }^{26}$. Embora não tenha se observado associação significativa entre sobrepeso e a presença de DCV no presente estudo, os riscos relacionados à obesidade são conhecidos na literatura e podem estar relacionados direta e indiretamente com a saúde cardiovascular ${ }^{4}$.

A atuação frente aos fatores comportamentais, não só relacionados ao tabagismo, mas também no consumo moderado de álcool, na adoção de uma dieta saudável e na redução da obesidade, constitui um importante campo de intervenção em saúde pública para manutenção da saúde e prevenção de doenças crônicas ${ }^{27}$. Dessa forma, os achados do presente estudo enfatizam a estreita 
relação entre os fatores de risco modificáveis e a presença de doenças cardiovasculares em idosos.

A presença de comorbidades, mais especificamente diabetes e hipertensão arterial, também apresentou associação estatisticamente significativa com as doenças cardiovasculares em idosos. Nesse contexto, o controle e prevenção de doenças como a diabetes e a hipertensão são importantes não só para a melhoria da condição de vida dos indivíduos, mas também para a diminuição da carga de doenças cardiovasculares na população idosa ${ }^{28}$, especialmente se consideradas as desigualdades existentes no município de São Paulo, que são fatores reconhecidamente associados à presença de doenças crônicas ${ }^{29,30}$.

Embora sejam escassos os estudos que analisaram a mudança na presença de doenças cardiovasculares na última década, a prevalência observada no início do segmento (2000) apresentou resultados semelhantes aos descritos por Lima-Costa et al. ${ }^{31}$, em uma análise dos dados da PNAD. E a ausência de diferenças significativas na prevalência de DCV, observada entre 2006 e 2010, apresentou o mesmo comportamento relatado na comparação entre 2003 e 2008, para a morbidade de doenças crônicas na população idosa brasileira ${ }^{29}$.

Em relação a 2010, a prevalência de doenças cardiovasculares nos idosos do estudo apresentou valores superiores quando comparados à estimativa da presença da doença na população idosa brasileira segundo a Pesquisa Nacional de Saúde, realizada em $2013^{22}$. Contudo, a comparação de indicadores de saúde entre regiões de grandes diferenças contextuais deve ser feita com cautela, visto a influência que as características do local de residência podem exercer nos desfechos de saú$\mathrm{de}^{32-34}$, sendo recomendados novos estudos que considerem as características contextuais para analisar as diferenças na prevalência de DCV.

A análise da diferença da presença de DCV ao longo do período possibilitou observar um aumento estatisticamente significativo de doenças cardiovasculares em idosos na última década. Em relação aos valores observados em 2000, verificou-se prevalências consistentemente mais elevadas em 2006 e 2010, não sendo observadas diferenças significativas entre as prevalências de
2010 em relação a 2006. Embora a mortalidade por DCV tenha diminuído nas últimas décadas ${ }^{35}$, o mesmo não se pode afirmar a respeito de sua morbidade, considerada uma crescente preocupação em saúde pública e apontada por Mathers et al. ${ }^{36}$ como a principal causa de mortes evitáveis em países da América Latina e Caribe, em 2011.

A morbidade por doenças cardiovasculares, especialmente na população idosa, é um tema emergente no campo do cuidado e planejamento em saúde. Responsável pelo maior número de anos vividos com incapacidade ${ }^{3}$ e líder de causas de morte evitáveis ${ }^{36}$, o aumento geral da prevalência de doenças cardiovasculares na população idosa, verificado no presente estudo, representa um importante desafio em saúde pública, principalmente se levado em consideração o rápido processo de envelhecimento recentemente observado na população brasileira ${ }^{37,38}$.

Este estudo apresenta algumas limitações a serem consideradas. Em primeiro lugar, a obtenção dos dados por entrevistas respondidas por idosos deve ser utilizado com cautela, uma vez que é possível a existência de vieses de memória nesse grupo populacional. Em segundo lugar, a característica do estudo seriado não possibilita a identificação de efeitos causais dos fatores individuais, que devem ser interpretados apenas como associações. Em terceiro lugar, a prática de atividade física pode atuar como um fator de confusão, mas a sua coleta não foi realizada de forma sistemática nas três ondas do estudo, impossibilitando a sua inclusão nos modelos. Em quarto lugar, as informações utilizadas sobre o consumo de álcool por idosos estão relacionadas ao autorrelato de ingestão nos três meses anteriores à entrevista domiciliar, não sendo possível a avaliação da quantidade específica de álcool ingerida pelos idosos.

Os resultados do presente estudo indicam um aumento geral da morbidade por doenças cardiovasculares em idosos no município de São Paulo na última década, bem como a importância dos fatores de risco modificáveis relacionados à DCV, e sugerem a necessidade do aumento de políticas de cuidado à saúde do idoso e atenção às formas de controle e prevenção relacionadas à maior causa de mortalidade na população idosa. 


\section{Colaboradores}

KHC Massa trabalhou na concepção do trabalho, análise e interpretação dos dados, revisão crítica e redação final do artigo, YAO Duarte trabalhou na concepção do projeto principal e na revisão crítica e ADP Chiavegatto Filho trabalhou na metodologia, interpretação dos dados, revisão crítica e redação final do artigo.

\section{Referências}

1. Global Burden of Disease Study 2013 Collaborators. Global, regional, and national incidence, prevalence, and years lived with disability for 301 acute and chronic diseases and injuries in 188 countries, 1990-2013: a systematic analysis for the Global Burden of Disease Study 2013. Lancet 2015; 386(9995):743-800.

2. Crimmins EM, Hayward MD, Hagedorn A, Saito Y, Brouard N. Change in disability-free life expectancy for Americans 70-years-old and older. Demography 2009; 46(3):627-646.

3. Prince MJ, Wu F, Guo Y, Gutierrez Robledo LM, O'Donnell M, Sullivan R, Yusuf S. The burden of disease in older people and implications for health policy and practice. Lancet 2015; 385(9967):549-562.

4. World Health Organization (WHO). Global atlas on cardiovascular disease prevention and control. Geneva: WHO; 2011.

5. World Health Organization (WHO). WHO methods for life expectancy and healthy life expectancy. Geneva: WHO; 2014.

6. GBD 2013 Mortality and Causes of Death Collaborators. Global, regional, and national age-sex specific all-cause and cause-specific mortality for 240 causes of death, 1990-2013: a systematic analysis for the Global Burden of Disease Study 2013. Lancet 2015; 385(9963):117-171.

7. Mathers CD, Fat DM, Boerma JT. The global burden of disease: 2004 update. WHO; 2008.

8. Brasil. Ministério da Saúde (MS). Informações de Saúde. Estatísticas Vitais. Brasília: MS; 2016.

9. Mansur AP, Favarato D. Mortalidade por Doenças Cardiovasculares no Brasil e na Região Metropolitana de São Paulo: Atualização 2011. Arq Bras Cardiol 2012; 99(2):755-761.

10. Schmidt MI, Duncan BB, Silva GA, Menezes AM, Monteiro CA, Barreto SM, Chor D, Menezes PR. Chronic non-communicable diseases in Brazil: burden and current challenges. Lancet 2011;377(9781):1949-1961.

11. Bhatnagar P, Wickramasinghe K, Wilkins E, Townsend $\mathrm{N}$. Trends in the epidemiology of cardiovascular disease in the UK. Heart 2016; 102(24):1945-1952.

12. Bauer UE, Briss PA, Goodman RA, Bowman BA. Prevention of chronic disease in the 21st century: elimination of the leading preventable causes of premature death and disability in the USA. Lancet 2014; 384(9937):45-52.

13. Theme Filha MM, Souza Júnior PRB, Damacena GN, Szwarcwald CL. Prevalência de doenças crônicas não transmissíveis e associação com autoavaliação de saúde: Pesquisa Nacional de Saúde, 2013. Rev Bras Epidemiol 2015; 18(Supl. 2):83-96.

14. Lebrão ML, Laurenti R. Saúde, bem-estar e envelhecimento: o estudo SABE no município de São Paulo. Rev Bras Epidemiol 2005; 8(2):127-141.

15. Brasil. Ministério da Saúde (MS). Protocolos do Sistema de Vigilância Alimentar e Nutricional - SISVAN na assistência à saúde. Brasília: MS; 2008.

16. Victora CG, Huttly SR, Fuchs SC, Olinto MTA. The role of conceptual frameworks in epidemiological analysis: a hierarchical approach. Int J Epidemiol 1997; 26(1):224-227. 
17. Rodriguez G, Goldman N. An assessment of estimation procedures for multilevel models with binary responses. J R Stat Soc Series A Stat Soc 1995; 158(1):73-89.

18. Niccoli T, Partridge L. Ageing as a risk factor for disease. Current Biology 2012; 22(17):R741-R752.

19. Siegel J. The demography and epidemiology of human health and aging. New York: Springer; 2012.

20. World Health Organization (WHO). World report on ageing and health. Geneva: WHO; 2015.

21. Camarano AA, Kanso S. Perspectivas de crescimento para a população brasileira: velhos e novos resultados. Rio de Janeiro: Ipea; 2009.

22. Instituto Brasileiro de Geografia e Estatística (IBGE). Pesquisa Nacional de Saúde 2013: percepção do estado de saúde, estilos de vida e doenças crônicas - Brasil, Grandes Regiões e Unidades da Federação. Rio de Janeiro: IBGE; 2014.

23. Barnett K, Mercer SW, Norbury M, Watt G, Wyke S, Guthrie B. Epidemiology of multimorbidity and implications for health care, research, and medical education: a cross-sectional study. Lancet 2012; 380(9836):37-43.

24. Newman A, Cauley JA. The epidemiology of aging. New York: Springer; 2012.

25. Ronksley PE, Brien SE, Turner BJ, Mukamal KJ, Ghali WA. Association of alcohol consumption with selected cardiovascular disease outcomes: a systematic review and meta-analysis. BMJ 2011; 342:d671.

26. Imes CC, Burke LE. The obesity epidemic: the USA as a cautionary tale for the rest of the world. Current Epidemiology Reports 2014; 1(2):82-88.

27. World Health Organization (WHO). Good health adds life to years: Global brief for World Health Day 2012. Geneva: WHO; 2012.

28. Mendis S, Chestnov O. The global burden of cardiovascular diseases: a challenge to improve. Current cardiology reports 2014; 16(5):1-9.

29. Barros MBA, Francisco PMSB, Zanchetta LM, César CLG. Tendências das desigualdades sociais e demográficas na prevalência de doenças crônicas no Brasil, PNAD: 2003-2008. Cien Saude Colet 2011; 16(9):37553768.

30. Malta DC, Bernal RIT, Almeida MCM, Ishitani LH, Girodo AM, Paixão LMMM, Oliveira MTO, Pimenta Junior FG, Silva Júnior JB. Desigualdades intraurbanas na distribuição dos fatores de risco para doenças crônicas não transmissíveis, Belo Horizonte, 2010. Rev Bras Epidemiol 2014; jul-set:629-641.
31. Lima-Costa MF, Barreto SM, Giatti L. Condições de saúde, capacidade funcional, uso de serviços de saúde e gastos com medicamentos da população idosa brasileira: um estudo descritivo baseado na Pesquisa Nacional por Amostra de Domicílios. Cad Saude Publica 2003; 19(3):735-743.

32. Kondo N, van Dam RM, Sembajwe G, Subramanian SV, Kawachi I, Yamagata Z. Income inequality and health: the role of population size, inequality threshold, period effects and lag effects. J Epidemiol Community Health 2012; 66(6):e11.

33. Chiavegatto Filho ADP, Lebrão ML, Kawachi I. Income inequality and elderly self-rated health in São Paulo. Ann Epidemiol 2012; 22(12):863-867.

34. Massa KHC, Pabayo R, Lebrão ML, Chiavegatto Filho ADP. Environmental factors and cardiovascular diseases: the association of income inequality and green spaces in elderly residents of São Paulo, Brazil. BMJ Open 2016; 6(9):e011850.

35. Araújo F, Gouvinhas C, Fontes F, La Vecchia C, Azevedo A, Lunet N. Trends in cardiovascular diseases and cancer mortality in 45 countries from five continents (1980-2010). Eur J Prev Cardiol 2014; 21(8):1004-1017.

36. Mathers CD, Stevens GA, Boerma T, White RA, Tobias MI. Causes of international increases in older age life expectancy. Lancet 2015; 385(9967):540-548.

37. Instituto Brasileiro de Geografia e Estatística (IBGE). Projeção da população do Brasil por sexo e idade, 1980 2050: revisão 2013. Rio de Janeiro: IBGE; 2013.

38. Carvalho JAM, Garcia RA. O envelhecimento da população brasileira: um enfoque demográfico. Cad Saude Publica 2003; 19(3):725-733.

Artigo apresentado em 07/12/2016

Aprovado em 30/03/2017

Versão final apresentada em 01/04/2017 